\title{
MedienPädagogik
}

Zeitschrift für Theorie und Praxis der Medienbildung

Themenheft Nr. 39: Orientierungen in der digitalen Welt Herausgegeben von Bardo Herzig, Tilman-Mathies Klar, Alexander Martin und Dorothee M. Meister

\section{Zur Bedeutung des Nichtwissens und die Suche nach Problemlösungen}

\author{
Bildungstheoretische Überlegungen zur Künstlichen Intelligenz \\ Claudia de Witt und Christian Leineweber
}

\section{Zusammenfassung}

Als disruptive Technologie verändert und beeinflusst Künstliche Intelligenz fast alle menschlichen Lebensbereiche und stellt das Bild und die Bildung des Menschen auf die Probe. Dabei stellt gerade das Nichtwissen ein bedeutendes Unterscheidungsmerkmal zwischen Mensch und Maschine dar und treibt den Menschen in unsicheren Situationen zu Problemlösungen und Urteilen an. Diese Fähigkeiten machen die Einzigartigkeit des Menschen aus und bleiben auch in Zukunft eine wichtige Bildungsaufgabe. Medienpädagogik nimmt in dieser Konstellation eine Aufklärungs- und Gestaltungsfunktion für die Verbindung zwischen dem individuell bzw. sozial Kontingentem und den materiellen, technischen Umwelten ein. Im vorliegenden Beitrag werden diese Überlegungen anhand interdisziplinärer Theorieansätze begründet.

The relevance of not knowing and the search for solutions to problems. Theoretical Considerations on Artificial Intelligence

\begin{abstract}
As a disruptive technology, artificial intelligence changes and influences almost all areas of human life and puts the image and education of human beings to the test. Not knowing is a significant differentiator between human beings and machinery and drives people to solve problems and make judgments in uncertain situations. These skills make humans unique and will continue to be an important educational task in the future. Media education assumes to be an educational function for the connection between the individual or social contingent and the material, technical environment. This article justifies these considerations on the basis of interdisciplinary theoretical approaches.
\end{abstract}




\section{Einleitung}

Künstliche Intelligenz stellt das Bild vom Menschen und seiner Bildung auf die Probe. Wird die menschliche Biologie zu einer posthumanen umgestaltet? Wird eine maschinelle Intelligenz die biologische Intelligenz übertreffen (vgl. Bostrom 2018)? Wird der Mensch in seinem Denken, Lernen, Handeln und Urteilen von maschinellen Berechnungen abgelöst? Ziel der vorliegenden Überlegungen ist die medienpädagogische Reflexion dieser Probe. Es wird einerseits eine bildungswissenschaftliche Orientierung zur Einschätzung des Phänomens der Künstlichen Intelligenz gegeben. Andererseits ist darauf zu blicken, welche Orientierung die Medienpädagogik der Gesellschaft geben muss, wenn sie eine der Künstlichen Intelligenz oftmals nachgesagte «Desillusionierung des menschlichen Selbstbildes» (Krämer 1997, 96) nicht billigend in Kauf nehmen und weiterhin für die Ermöglichung von Bildung einstehen will.

Ausgehend von einer Deutung des Phänomens der Künstlichen Intelligenz als disruptive Intelligenz, die eine Kybernetisierung des gesellschaftlichen Lebens intendiert, fussen die folgenden Überlegungen auf einer bildungstheoretischen Perspektive, die Bildung ausgehend vom Sozialen denken lässt. Leitend dafür steht die These, dass Bildung die Aneignung sozial tradierter Verhaltensweisen und Kenntnisse voraussetzt, auf deren Basis Subjekte die Fähigkeit des eigenen Handelns und Urteilsvermögens ausbilden. Es ist eine These, die in Anbetracht maschineller Möglichkeiten auch dann zukünftig aufrechterhalten werden kann, wenn die Menschheit nicht von einem selbstgeschaffenen technischen Machwerk beherrscht wird (vgl. Rohbeck 1993, 10), sondern weiterhin auf ihre wesensspezifischen Veranlagungen setzt und Maschinen in den Diensten eines sozial konstituierten Lebens (von Menschen) kontrolliert wirken lässt.

Unter diesen Bedingungen liegt ein entscheidender Impuls in dem Sachverhalt, dass man der Künstlichen Intelligenz gegenwärtig in erster Linie den Vorteil zuspricht, dass sie sicherer, schneller und beständiger auf der Grundlage von Daten mit Problemen rechnen kann (vgl. Baecker 2019, 16-39). Wenn der Mensch in seiner Einzigartigkeit durch diese Annahme vehement infrage gestellt zu sein scheint, dann wird dieser Infragestellung im Weiteren der Begriff des Nichtwissens entgegengebracht, den Luhmann als pädagogische Notwendigkeit ansah, um die Lernenden auf eine - im Zuge technischer Fortentwicklungen - zunehmend unbestimmt bleibende Zukunft einzustellen (vgl. Luhmann 2002, 198). Nichtwissen heisst folglich: das Unbekannte zu akzeptieren, aber womöglich auch zu nutzen, um im Modus der Kontingenz Entscheidungen treffen und Probleme lösen zu können. Für die Überlegungen des vorliegenden Beitrags ist dabei vor allem Luhmanns These entscheidend, Maschinen seien durchaus in der Lage, eine bewusste, symbolverarbeitende Intelligenz zu erreichen, während ihnen der Umgang mit Nichtwissen, d.h. der Umgang mit Symbolen, die noch nicht bekannt sein können, verwehrt bleiben müsse (vgl. Luhmann 2002). 
Mit Luhmann ist folglich eine Position gefunden, die den Menschen positiv von der Maschine unterscheidet. Eine gewichtige Folge dieser Position liegt in der Notwendigkeit einer gleichsam handlungs- wie urteilsbezogenen Problemlösungsfähigkeit des Subjekts innerhalb einer von Künstlicher Intelligenz durchwirkten Lebenswelt. In Bezug auf das handelnde und urteilende Subjekt ist eine solche Fähigkeit Gabe und Bürde zugleich, weil mit jeder Problemlösung der Zugang zu neuen Problemen geebnet ist und weil mit jeder Form des Wissens auch darüber Gewissheit erlangt werden kann, was man noch nicht weiss. Das führt letztlich zu Überlegungen darüber, wie der Unterschied zwischen menschlicher und künstlicher Intelligenz in einem medienpädagogischen Rahmen als bildungsermöglichende Relation genutzt werden kann. Medienpädagogik kann in diesem Sinne als orientierungsgebende Disziplin verstanden werden, die eine Aufklärungs- und Gestaltungsfunktion für die Verbindung zwischen dem individuell bzw. sozial Kontingenten und den materiellen, technischen Umwelten einnimmt.

\section{Künstliche Intelligenz als disruptive Technologie und die fortschreitende Kybernetisierung}

Entwicklungsgeschichtlich ist Künstliche Intelligenz schon in der Antike vorzufinden (vgl. Görtz und Nebel 2014, 5). Görz und Nebel machen drei Entwicklungslinien dafür verantwortlich, die dazu führten, dass Künstliche Intelligenz ihr heutiges Potenzial erreichen konnte: (1) der seit Jahrhunderten existierende Mythos von der Erzeugung künstlicher Menschen, (2) die Idee von der «Maschinisierung menschlicher Verstandesleistungen, deren «Ursprung in der kulturellen Entwicklung der Schriftsprachlichkeit, insbesondere des schriftlichen Rechnens» liegt, und (3) «die Erfindung des klassischen programmierbaren Universalrechners» (ebd.). Künstliche Intelligenz ist zunächst eine Disziplin in der Informatik, die sich mit der Automatisierung intelligenten Verhaltens beschäftigt und als deren Gründungsvater der Mathematiker Alan Turing, mit seinem 1950 erschienenen Werk Computing Machine and Intelligence, angesehen werden kann (vgl. ebd.). An Turing anschliessend wurden die Möglichkeiten zur Simulation menschlicher Wahrnehmungs- und Verstandesleistungen mit dem Sprachlichen immer weiter ausgebaut:

«Ein entscheidender Aspekt, warum der Computer zum zentralen Mittel der Forschung werden konnte und damit KI erst möglich wurde, liegt in der grundlegenden Neuerung, dass er ein Maschinentyp ist, der Sprache verwendet. Auch wenn es sich dabei erst einmal um formale Sprachen zur Programmierung von Automaten handelt, stand schon immer die Absicht dahinter, die Kluft zwischen umgangssprachlich oder fachsprachlich formulierten Aussagen und ihrer formalsprachlichen Umsetzung möglichst eng zu machen. Die Fähigkeit, kompetent zu sprechen, schließt aber auch das Vermögen zu urteilendem 
Denken ein. Daher warf die maschinelle Verwendung der Sprache von Anfang an die Frage auf, ob Maschinen nicht im Prinzip auch denken könnten - wobei das Denken aber immer schon als die Fähigkeit galt, die den Menschen auszeichnet.» (ebd., 8)

Aus dieser Frage hat sich die Kontroverse zwischen schwacher und starker Künstlicher Intelligenz entwickelt: «Während die schwache KI-Hypothese mit der Werkzeugperspektive auf Systeme der KI verträglich ist, will die starke entscheidend darüber hinausgehen.» (ebd., 8f.) Bei einer schwachen Künstlichen Intelligenz werden verschiedene Methoden eingesetzt, die dazu beitragen, dass intelligentes Verhalten simuliert wird und eine Maschine solche Aufgaben ausführen kann, die mit kognitiven Leistungen verbunden werden (vgl. Searle 1980, 2). Zum intelligenten Verhalten von Maschinen gehören dann Möglichkeiten zur Wahrnehmung, Fähigkeiten zum logischen Schlussfolgern, Fähigkeiten zur Simulation selbstständigen Lernens und zum eigenständigen Finden von Problemlösungen. Eine schwache Künstliche Intelligenz kann uns im Kontext von Bildung helfen zu verstehen, wie wir unsere menschlichen kognitiven Fähigkeiten verbessern und weiterentwickeln können und wie unser Lernen funktioniert. Künstliche Intelligenz kann nicht sich selbst verstehen oder Entscheidungen rechtfertigen und verfügt nicht über die Wahrnehmung einer Selbstwirksamkeit (Self-Awareness), die besonders bei erfolgreichen Lernenden zu erkennen ist, weil diese nämlich genau beurteilen können, was sie wissen und was sie ausmacht; aber gerade die Kombination aus grossen Datenmengen und gut gestalteter Künstlicher Intelligenz kann uns nach Luckin dabei helfen, die Art und Weise zu verfolgen, in der sich unsere Intelligenz und unsere Lernfortschritte entwickeln (vgl. Luckin 2018, 91). Eine starke Intelligenz liegt vor, wenn Systeme so etwas wie Bewusstsein entwickeln und wenn letztlich dieses maschinelle Bewusstsein dem menschlichen ebenbürtig oder sogar überlegen ist (vgl. Searle 1980, 2), wovon die bisherigen Systeme aber noch weit entfernt sind. Bis jetzt ist daher menschliche Intelligenz doch wesentlich mehr: die Fähigkeit, sein Denken bewusst auf neue Herausforderungen einzustellen und neue Situationen zu bewältigen. Menschliche Intelligenz ist die Fähigkeit, sich in neuen Situationen zurecht zu finden und dabei geistig anpassungsfähig an neue Aufgaben zu sein.

Nichtsdestotrotz ist Künstliche Intelligenz bereits als eine disruptive Technologie anzusehen. Zwar haben für Mersch die technischen Erfindungen wie Telefon, Kinematografie oder Fernsehen zur Entstehung einer digitalen Kultur beigetragen, aber ausschlaggebend für die disruptive Entwicklung sei einerseits «die Miniaturisierung des Computers» in den 1970er Jahren gewesen, «die wiederum auf Demokratisierungsideen der 1960er Jahre beruhte», und andererseits der Personal Computer «als eine Apparatur der Befreiung und Gegenoffensive gegen staatliche Kontrolle» (Mersch 2017, o.S.). Disruptiv bezeichnet man eine Technologie folglich dann, wenn sie etwas 
bisher erfolgreich Bestehendes zerstört, obsolet macht, ersetzt oder vollständig verdrängt. Und dabei handelt es sich nicht nur um Technologien, sondern um technologiebasierte Produkte und Verfahren, die beispielsweise die Märkte, aber auch eben unser Verhalten enorm beeinflussen. Künstliche Intelligenz ist dabei, nicht nur ganze Branchen, sondern alle Bereiche unseres menschlichen Lebens zu durchdringen und zu verändern. Autonome Gegenstände wie Roboter oder selbstfahrende Autos sollen uns Menschen von routinemässigen oder gefährlichen Tätigkeiten entlasten, lernende Maschinen werden nicht nur Routinetätigkeiten übernehmen, sondern auch Entscheidungen treffen und Diagnosen stellen.

Eine verstärkende Dimension der Disruption, der Zerstörung oder Verdrängung, nimmt Künstliche Intelligenz noch einmal dann ein, wenn man sie aus einer anthropologischen und technikphilosophischen Perspektive betrachtet. So vertritt beispielsweise der technologische Posthumanismus die Position, Künstliche Intelligenz sei insofern disruptiv, als dass sie den Status des Menschen als Krone der Schöpfung infrage stelle und eine maschinelle Superspezies (vgl. Loh 2018) schaffe. Der technologische Posthumanismus geht zwar gemäss Janina Loh davon aus, dass der Mensch von den technologischen Errungenschaften profitiere und «zu einer weitaus besseren Version seiner selbst» werde; vor allem erschaffe er aber eine artifizielle «Alterität - Andersartigkeit, Verschiedenheit, die die menschliche Spezies ablöst und damit 〈den〉 Menschen überwindet» (ebd., 12f.). Damit ist Technik für den technologischen Posthumanismus Ziel und Zweck. Eher ein Mittel zum Zweck ist die Technik für den Transhumanismus, um den Menschen weiter zu entwickeln, zu optimieren, zu modifizieren und zu verbessern. «Die Technik spielt im transhumanistischen Denken die Rolle des Mediums und Mittels zum Zweck der Optimierung des Menschen zu einem Menschen x.0» (ebd., 11).

Mit den hier skizzierten Positionen drohen der deutsche Idealismus und Neuhumanismus, welche die natürliche Vernunftbegabung und Rationalität als Kernkompetenzen des eigenverantwortlichen und autonomen Menschen auslegten (vgl. ebd., 20) und auf diese Weise Ideale wie Selbstbestimmung, freie Persönlichkeitsauffassung und ethische Normen starkmachten, obsolet zu werden oder sich langsam aufzulösen. Nach der Einführung der skriptografischen Datenverarbeitung vor ca. 2.500 Jahren (materielle Gegenstände wie Stein, Ton, Papier), nach der Einführung des Buchdrucks vor ca. 560 Jahren oder mit der Einführung der elektronischen Medien vor mehr als 100 Jahren schreitet mit Künstlicher Intelligenz die Kybernetisierung fort und scheint «ein technologisches Modell von Subjektivierung und auch des Unbewussten» stak zu machen (Hörl 2011, 33). 


\section{Künstliche Intelligenz und das Problem der Objektivierung des Sozialen}

Eine zentrale Herausforderung, die Künstliche Intelligenz an die Medienpädagogik stellt, liegt nun darin, dass man zwar verstehen kann, was als intelligent rechnende Technik funktioniert, jedoch zumeist unklar bleibt, vor welchem Hintergrund und mit welchen Intentionen sie eigentlich funktioniert. Das damit konsequent verbundene Misstrauen der Medienpädagogik an der Künstlichen Intelligenz als black box ist auch (oder gerade) in Bezug auf die Bildung des Menschen keineswegs neu. So legte beispielsweise Sesink bereits 1990 mit dem Beitrag «Künstliche Intelligenz», Systemreproduktion und Bildung eine kritische Auseinandersetzung mit der Vision vor, dass autonom operierende Maschinen dem Menschen eines Tages «das Denken, das Lernen und die Kreativität» (Sesink 1990, 193) abnehmen könnten. Bisher einzigartige menschliche Fähigkeiten werden auf diese Weise maschinell objektiviert. Im Folgenden geht es um eine detaillierte Rezeption dieser kritischen Auseinandersetzung, die ein bildungstheoretisches Fundament aufbaut, von dem eine Hinführung zu einer Orientierung gebenden Medienpädagogik geleistet werden kann.

Sesink hält eine Auseinandersetzung mit der Vision kybernetischer, autonom rechnender Maschinen insofern für relevant, als sie in erster Linie intendiert, «daß es für die Sicherung des Fortbestands einer Gesellschaft in Zukunft weder unumgänglich ist, daß Menschen entsprechende Qualifikationen erwerben, noch die Pädagogik weiterhin gefordert ist, den objektiven Gesellschaftszusammenhang zu rekonstruieren» (ebd., 195).

Die zwei wichtigsten Thesen Sesinks sind damit bereits angedeutet: Künstliche Intelligenz zeichnet einerseits ein Bild vom Menschen, das die menschliche Einzigartigkeit, die Wesenszüge des Subjekts, das Humane nach und nach verliert, was gleichsam ein Bild von einer intelligenten Maschine als ein Objekt ohne jegliche Individualität impliziert (vgl. ebenso Sesink 2012, o.S.). Dies heisst vor allem, dass die "geschichtliche Bedeutung von Bildung» infrage gestellt wird, «und zwar in den beiden Dimensionen der System-Reproduktion und der geschichtlichen Kontinuität» (Sesink 1990, 194). Wer auf diese Entwicklung (medien-)pädagogisch reagieren will, muss daher andererseits die gesellschaftliche Funktion der Künstlichen Intelligenz hinterfragen, die sich aus bildungstheoretischer Perspektive mit der «Frage nach der Bedeutung des Individuellen für das Gesellschaftliche» (ebd., 195) einholen lässt.

Das Potenzial Künstlicher Intelligenz, das Subjekt in seinen Denk- und Lernfähigkeiten zu überholen oder sogar zu ersetzen, kommt nach Sesink von der Fähigkeit der kybernetischen Maschinen, «System-Strukturen und ihre Funktionsbeziehungen perfekter abzubilden, als es irgendein individueller Menschenkopf zu leisten vermöchte» (ebd., 194). Dies legt Sesink anhand der «biokybernetischen Simulation von Lebenskreisläufen» und der sozialkybernetischen Simulation von gesellschaftlichen Systemprozessen» (ebd., 196ff.) dar. Die Perspektiven auf Bio- und Sozialsysteme 
dienen Sesink dazu, das Verhältnis zwischen Welt, Mensch und Gesellschaft bzw. zwischen Objektivität, Subjektivität und Intersubjektivität zu bestimmen. «Das Biosystem ist nicht von Menschen gemacht. Sozialsysteme hingegen sind ein Produkt menschlicher Praxis.» (ebd., 199) Das Biosystem existiert folglich auf der Basis objektiv gegebener Naturzusammenhänge, während Sozialsysteme die Produkte intersubjektiver Interpretationen und Abstraktionen ebendieser Zusammenhänge sind. Das Biosystem ist die Grundlage des menschlichen Lebens, während Sozialsysteme die Subjekte an bestehende Zusammenhänge binden, in denen sie leben und aus denen heraus sie handeln und urteilen. Subjekte befinden sich daher stets in sozialen Strukturen, die ihr Handeln und Urteilen fundieren.

Dieser systemischen Rahmung des subjektiven und sozialen Lebens begegnet Sesink nun mit einer bildungstheoretischen Radikalität, die den Subjekten ein "grundsätzlich revolutionäres Potential» (ebd., 200) unterstellt. Subjekte sind in diesem Sinne nur dann bereit, die Strukturen der sozialen Systeme, in denen sie handeln und urteilen, zu akzeptieren, sofern ihre Lebensverhältnisse in ihnen «gut aufgehoben sind» (ebd.), woraus folgender Anspruch an subjektives Leben erhoben werden kann:

«Nicht die Systemreproduktion, sondern ihre Lebensreproduktion ist ihnen [den Subjekten, CdW \& CL] oberstes Ziel. Nur bis zu einem, für sie mit Sicherheit vorhersagbaren Punkt nehmen sie Verstöße gegen Lebensinteressen hin; ist dieser Punkt überschritten, droht dem System Aufkündigung der Loyalität.» (ebd.)

Wenn damit also das emanzipatorische Potenzial der Subjekte in einer Kritik am Sozialen bzw. an den Strukturen sozialer Systeme liegt, dann ist eine Schwierigkeit der Künstlichen Intelligenz schliesslich darin auszumachen, dass sie eine Loslösung des Systemischen aus der Abhängigkeit subjektiver Akzeptanz intendiert (vgl. ebd., 201). Künstliche Intelligenz bedeutet demzufolge Objektivierung des Sozialen, indem sie (inter-)subjektiv Validiertes über maschinelle Berechnungen abstrahiert, sodass ein «automatisches System entsteht», das als eine «selbstständige, von der menschlichen Subjektivität abgelöste, ‘absolute) Existenz» (ebd.) agiert.

Die zentrale Problematik, die daraus in bildungstheoretischer Hinsicht resultiert, ergründet Sesink mit dem «Recht des Allgemeinen» (ebd., 202), eine theoretische Figur, die auf die Philosophie Hegels zurückführt und besagt, dass das Projekt der Bildung nur dann gelingen kann, wenn sich das Subjekt zur Gesellschaft und ihren Bestimmungen verhält (vgl. Hegel 1821/2015, 16ff.). Bildung ist damit ganz grundsätzlich auf der Basis des Verhältnisses zwischen Subjekt und sozialer Welt bestimmt (vgl. Sesink 1990, 202). Sie setzt in diesem Sinne eine Aneignung sozialer Verhältnisse und Kenntnisse voraus, die es erst möglich werden lässt, dass Subjekte eine «Fähigkeit zur Selbstbestimmung in der Welt zu bilden vermögen» (ebd., 203). Man weiss 
demnach erst, wie zu handeln und zu urteilen ist, wenn man weiss, wie schon gehandelt und geurteilt wird (vgl. Menke 2018, 30). Hegel geht es damit um das subjektive Erlangen von Handlungsfähigkeit im sozialen System. Die Bildung des Einzelnen ist stets in eine Gesellschaft eingewoben. Die Grundidee einer so verstandenen Bildung liegt folglich darin, dass sich immer zwei differente Seiten gleichzeitig begegnen: objektive Allgemeinheit und subjektive Individualität (vgl. Sesink 1990, 202) bzw. soziale Gesetzmässigkeit und individuelle Freiheit.

Dieser Idee ist im Grunde genommen eine stete Reproduktion sozialer Systemzusammenhänge implizit. Die Aneignung sozialer Verhältnisse und Kenntnisse setzt ein Wissen voraus, dass Handlungen in einem bestimmten System andere Reaktionen nach sich ziehen können als in anderen Systemen. Bereits Kinder entwickeln recht zeitnah ein Gespür dafür, dass dasselbe Verhalten im Sozialsystem «Freundeskreis` zu Anerkennung und im Sozialsystem ‘Familie` zu Tadel führen kann (vgl. Jörissen und Marotzki 2009, 23). In der Welt zu handeln und zu urteilen heisst somit immer auch, die eigenen Grenzen auszuloten. Einerseits ist dies für den gesellschaftlich an die Pädagogik gerichteten Auftrag der Erziehung mit der nüchternen Einsicht verbunden, dass Lernende niemals ausschliesslich als Trivialmaschinen betrachtet werden dürfen, weil ihre Reaktionen auf die Sozialsysteme der Welt immer durch eine Selbstreferenz in Form eigener Erfahrungen, Befindlichkeiten oder Affekte ermittelt werden (vgl. Luhmann 1984, 15; Luhmann 2002, 79). Andererseits ist es gerade die damit einhergehende Unzuverlässigkeit, die das Subjekt unter Verantwortung stellt. Erst durch Selbstreferenz wird das, was in der Welt erfahrbar ist, zur Grundlage des eigenen Seins. Im Einklang dazu steht die Grundidee zeitgenössischer Bildungstheorien, den Ursprung von Bildungsprozessen auf solche Krisenerfahrungen zurückzuführen, in denen Subjekte mit Problemlagen konfrontiert werden, zu deren Bewältigung sich bislang angeeignete Welt- und Selbstverständnisse als nicht mehr ausreichend erweisen (vgl. z.B. Marotzki 1990, 25; Koller 2012, 16). Die Selbstreferenz der Subjekte bestimmt das Wesen der Bildung nicht nur, indem sie angeeignete Handlungs- und Urteilsformen eine orientierende Gestalt gibt, sondern auch, indem sie die Souveränität des Einzelnen stets dadurch aufs Neue herausfordert, dass jede Herstellung von Sicherheit neue Bereiche der Unsicherheit öffnet (vgl. Jörissen und Marotzki 2009, 21). Auf den Punkt gebracht: Bildung lebt von der Gegebenheit, dass jede Selbstverortung in der Welt stets auch andere Verortungen zulässt.

Gegenüber dieser offenen «Entdeckungsreise» (Sesink 1990, 204) des Subjekts verhalten sich maschinelle Berechnungen prinzipiell konträr. Schreibt man nämlich der Künstlichen Intelligenz eine Objektivierung des Sozialen zu, dann muss man davon ausgehen, dass soziale Systemzusammenhänge durch berechnete Konstrukte verändert werden. Auf mögliche Konsequenzen dieser Veränderung in Bezug auf die Gesellschaft hat der soziologische Systemtheoretiker Dirk Baecker erstmals mit der These von der nächsten Gesellschaft hingewiesen (vgl. Baecker 2007), die auf 
der Idee fusst, dass sich zunehmend Maschinen auf Basis eigenständig operierender Algorithmen «an der Kommunikation unter Menschen» (Baecker 2018, 20) beteiligen, wodurch die funktionale Differenzierung der Moderne in ein hybrid kombiniertes Netzwerk überzugehen scheint (vgl. ebd., 26). Nun lässt sich gegen diese Veränderung auf der Ebene der Subjekte mit Sesink einwenden, dass maschinell konstruierte Netzwerkstrukturen auf einer Art selbstreferenzlosem Denken basieren: "Maschinelle Intelligenz besteht in den reinen Formen des Denkens, ohne daß diese noch einen Bezug zum Inhalt hätten.» (Sesink 1990, 205) Unter solchen Bedingungen werden in den Strukturen des Netzwerks subjektiv ermittelte und intersubjektiv vermittelte Zusammenhänge, Widerstände oder Erinnerungen durch eine maschinell vollzogene Ansammlung von Daten ersetzt. Dies gewährt zwar eine «Fortführung von Systemstrukturen in die Zukunft» (ebd., 206), allerdings ohne dem bildungstheoretischen Anspruch genügen zu können, dass der Antrieb subjektiver Handlungs- und Urteilsfähigkeit aus der Differenz zwischen dem, wie das Subjekt sich selbst in der Welt verortet, und dem, wie es sich gerne verorten würde. Eine ausschliesslich durch Künstliche Intelligenz gestaltete Form der Gesellschaft verzichtet demnach auf eine Stimulation durch die Bedürfnisse, Mängel oder Probleme, die aus der Auseinandersetzung der Subjekte mit ihren sozialen Verhältnissen resultieren. Sie ist somit unfähig, das menschliche Interesse an der Welt in sich aufzunehmen (vgl. ebd.).

In erster Linie und mit aller Deutlichkeit wird damit von Sesink ein drohender Bedeutungsverlust des biologischen Menschen moniert, der in einem «sozialkybernetisch regulierten gesellschaftlichen» System lediglich noch als Parasit «des Gesamtsystems» (ebd., 202) agieren könnte. Es ist ein Verlust, mit dem sich jedoch gleichsam die gesellschaftliche Rolle der (Medien-)Pädagogik stärken liesse, dann nämlich, wenn sie Impulse dafür liefern könnte, inwiefern die Menschheit auch zukünftig Maschinen, insbesondere automatisch operierende Maschinen «im Dienste ihres eigenen Lebens» (ebd., 206) wirken lassen könnte. Die Grundbedingungen einer solchen Impulsgabe gilt es im Folgenden zu ergründen.

\section{Intelligenz und die Bedeutung des Nichtwissens für Bildung}

In ihrem Beitrag Die Maschine - Materialität, Metapher, Mythos: Ethische Perspektiven auf das Verhältnis von Mensch und Maschine macht Thimm auf das Problem aufmerksam, dass technische Entwicklungen ihren gesellschaftlichen Reflexionen stets vorauseilen, wodurch die Durchdringungen der Künstlichen Intelligenz in alle Lebensbereiche dazu führen könne, dass der Mensch «nicht mehr gebraucht» werde (Thimm 2019, 36). Im Detail heisst das:

«Maschinen lernen aus Material, das Menschen (mehr oder weniger) freiwillig zur Verfügung stellen, sie modellieren aus kulturellen Artefakten wie Texten, Bildern oder Filmen, und aus den alltäglichen digitalen Spuren der Menschen 
umfassende Muster und Handlungstypen, die dann in den menschlichen Alltag zurückgespeist werden. Der Wissenskanon [...] fungiert nicht mehr nur als kulturelles Gut, um menschliches Wissen und Kultur für zukünftige Generationen zu bewahren, sondern als Trainingsdatenbank für Maschinen, die aus dieser menschlichen Kreativität lernen. Ziel ist dabei also nicht, dass [...] Menschen dauerhaft auf große Wissensbestände zugreifen können, sondern dass das Wissen der Menschheit die Maschinen klüger macht» (ebd.).

Sofern es die Kybernetik ist, die sich als Wissenschaft der Steuerung, Regelung und Kommunikation in Lebewesen und Maschinen widmet (vgl. Wiener 1963), stellt sie ein begriffliches Instrumentarium bereit, um das verstehen zu können, was gegenwärtig in innerhalb der Beziehung zwischen Mensch und Maschine unter dem Terminus der Künstlichen Intelligenz firmiert. Hier lässt sich zunächst der Verhandlungsgegenstand mit der Beobachtung Herbert A. Simons' eingrenzen, wonach Suchverfahren auf Basis maschinell berechneter Kombination dort sinnvoll und lebensweltlich kompatibel erscheinen, wo das Problem die Lösungskompetenz eines Einzelnen überschreitet (vgl. Simons 1995, 114ff.). Die Umsetzung dieses Potenzials erfolgt gegenwärtig anhand informationsbezogener und statistisch hergeleiteter Beurteilungen. In den Worten Baeckers heisst das:

«Künstliche Systeme sind im Moment Systeme, denen virtuelle Intelligenz insofern zugesprochen werden kann, als sie ohne den Zugriff auf große Datenmengen, in denen ihre Fähigkeit zur Mustererkennung und Schlussfolgerung trainiert und getestet werden kann, nicht vorstellbar sind. Maschinenlernen heißt in diesem Zusammenhang, Hypothesen zur Wahrscheinlichkeit des Auftretens von Ereignissen und Zusammenhängen zu entwickeln, die mit jedem neuen Fall und jedem neuen Datenset geprüft und korrigiert werden können» (Baecker 2019, 59).

Bereits in den 1960er-Jahren gab es in der Erziehungswissenschaft solche kybernetisch orientierten Ansätze, die auf die «Objektivation geistiger Prozesse mittels informationeller Systeme» vom Lernen, Erinnern bis hin zum Wertsetzen zielten (vgl. Pongratz 1978, 12). Im Hinblick auf einen sozial konstituierten Begriff von Bildung liegt die wesentliche Herausforderung beim Lösen von Problemen darin, dass sich Subjekte solche Strategien und Normen aneignen, die sich in ganz ähnlichen Fällen bereits als Handlungs- und Urteilshilfen bewähren konnten. Sofern man diese Deutung bejaht, lässt sie sich jedoch gleichsam mit Verweis auf die Sozialstrukturen moderner, technisch weit fortgeschrittener Gesellschaften durch das Phänomen der Kontingenz in Frage stellen. In einer sehr allgemeinen Bestimmung umfasst Kontingenz den Sachverhalt, dass jede getroffene Entscheidung nur eine Möglichkeit darstellt, die prinzipiell auch anders ausfallen könnte als man erwarten würde oder 
planen könnte: Etwas wird zwar als richtig anerkannt, scheint aber prinzipiell auch anders möglich (vgl. Luhmann 1971, 53). Implizit ist dieser Bestimmung eine angenommene Begrenzung des Wissens unterlegt, die sich massgeblich aus der Paradoxie heraus aufbaut, dass jede getroffene Sinnzuschreibung eine «Einteilung der Welt in eine bekannte Innenseite und eine unbekannte Außenseite» (Luhmann 1992, 168) intendiert. Subjektiv aufgeworfene Perspektiven unterliegen auf diese Weise stets einer zwingend notwendigen Unvollständigkeit, die offen lässt, ob etwas nicht auch anders gesehen werden könnte. Selbst die Individuen der sogenannten Wissensgesellschaft (vgl. Stehr 1994) können daher niemals eine vollständige Gewissheit darüber erlangen, wie weit ihr Denken im Hinblick auf die Gesamtheit der Welt reicht (vgl. Steiner 2006, 15).

Mit anderen Worten repräsentiert der Begriff der Kontingenz eine Struktur der subjektiven Sinnbildung (vgl. Rustemeyer 2011, 115), die als Reflexionsfigur dafür heranzuziehen ist, dass man mit der Potenzierung von Wissen immer weniger wissen kann. So ist es auch kein Zufall, dass populäre Zeitdiagnosen um die Jahrtausendwende ${ }^{1}$ im thematischen Horizont subjektiver Unsicherheit vermehrt vom Begriff des Nichtwissens sprechen (vgl. Wimmer 2014, 245). Gerade Luhmann sah in diesem Kontext die Notwendigkeit der Pädagogik darin, «den zu erziehenden Nachwuchs auf eine unbekannt bleibende Zukunft» einzustellen, womit «das Lernen von Wissen weitgehend ersetzt werden müsste durch das Lernen des Entscheidens, das heißt: des Ausnutzens von Nichtwissen» (Luhmann 2002, 198). Angesprochen ist damit der Anspruch eines proaktiven Umgangs mit dem inhaltlich Unbestimmten, der einen Weg zu geeigneten Problemlösungen ebnen soll. Luhmann gelangt zu dieser Einschätzung einerseits im Anschluss an Analysen zu den vermehrt auftretenden Umweltproblemen der Moderne, die ihn zu der These veranlassen, dass die Menschheit zwar immer mehr «ökologisches Wissen» sammle, aber auf diese Weise gleichsam mit einem ansteigenden «Nichtwissen über die Beziehungen zwischen der Gesellschaft und ihrer ökologischen Umwelt» (Luhmann 1992, 158) konfrontiert werde. Andererseits erkennt er für das gesellschaftliche Funktionssystem der Wissenschaft, dass Wissensgewinne stets «eine Reproduktion der forschungsnotwendigen Spezifikation» eines Nichtwissens nach sich ziehen, was sich empirisch in einer laufenden «Erneuerung spezifischer Problemstellungen» niederschlägt, «die auch akzeptiertes Wissen immer wieder in Frage stellen» (Luhmann 1995, 178).

Wenngleich diese Beispiele verdeutlichen, dass der Begriff des Nichtwissens mit einer Vielzahl an vielschichtigen sowie mehrdimensionalen Lesarten konfrontiert (vgl. Wehling 2006, 148), bietet seine theoretische Rahmung - die kontingente Struktur, das heisst inhaltliche Unbestimmtheit der Welt - eine Möglichkeit, um einen

1 So zum Beispiel Zygmunt Baumans Soziologie der Postmoderne, Ulrich Becks Theorie reflexiver Modernisierung oder Niklas Luhmanns Theorie funktional differenzierter Gesellschaftssysteme (vgl. Wehling 2006, 149-214). 
(zeitgemässen) Begriff von Intelligenz zu bestimmen, der sich mit Bezug auf den Bildungsbegriff als eine «Eigenschaft von Subjekten» (Sesink 1990, 195) verhandeln lässt und folglich eine Funktion bei der «Entwicklung neuer Bedeutungen und Pläne neuer Handlungen» (Dewey 1916/2004, 110) einzunehmen in der Lage ist. In diesem Rahmen und im Rückgriff auf das bislang Argumentierte lässt sich zunächst thesenartig formulieren: So wie Bildung die Aneignung sozialer Verhältnisse und Kenntnisse voraussetzt und Handlungs- und Urteilsfähigkeit stets ein Resultat von Problemlösungen ist, so geht Intelligenz davon aus, «daß von einem Nichtwissen auf ein Wissen geschlossen wird» (Baecker 2002, 57). Intelligenz im Kontext von Nichtwissen ist «die Form gewordene Vermutung» (ebd., 58).

In summa geht diesen Betrachtungen ein theoretischer Ort voraus, der in (der Überwindung) einer Differenz liegt. In diesem Sinne setzt Bildung die Differenz zwischen dem Subjekt und seinen sozialen Verhältnissen voraus, Handlungs- und Urteilsfähigkeit setzt die Differenz zwischen Problemen und ihren Lösungen voraus und Intelligenz setzt schliesslich die Differenz zwischen Wissen und Nichtwissen voraus. Die Beantwortung der Frage, wie nah diese Bestimmungen empirisch miteinander verknüpft sind, lässt sich in der Kybernetik beispielsweise bei Ashby $(1961 / 1981,295)$ finden, der eine formale Definition von Intelligenz zugrunde gelegt hat, die auf die Fähigkeit verweist, geeignete Lösungen für Probleme zu finden (vgl. darüber hinaus Baecker 2019, 9). Oder mit Luhmann formuliert: Intelligenz ist die Bezeichnung dafür, dass sich ein (menschliches) Lebewesen im Verhältnis zu sich selbst und seiner Umwelt für "die eine und nicht die andere Problemlösung» (Luhmann 1984, 158) entscheidet. Bemerkenswert ist dabei, dass Problemlösungen - neben der Wissensrepräsentation und -verarbeitung oder Kalkülen für automatisches Beweisen, Unsicherheit und Wahrscheinlichkeit - zu den zentralen Methoden der Künstlichen Intelligenz zählen (vgl. Görz und Nebel 2014, 42ff.).

Aus der Perspektive der Erziehungswissenschaftlerin Käte Meyer-Drawe (2005, 28ff.; 2007, 247) ist aber gerade ein Aushalten von Nichtwissen und der Unlösbarkeit von Problemen für die Bildung des Menschen unabdingbar, um letztlich zu plötzlicher Einsicht zu gelangen. Damit ist eine Beschreibung formuliert, mit der dem Bildungsbegriff eine Zeitstruktur zugewiesen wird, die gewöhnlichen, linear verlaufenden Zeitmustern entgegensteht. Die Bildung des Menschen lebt insofern von einer Prozessualität, die dadurch gekennzeichnet ist, dass Subjekte Erfahrungen machen, die von Unsicherheiten, Irritationen und Unterbrechungen leben (vgl. Leineweber 2020, 217-224). Derartige Erfahrungen lassen sich mit ausschliesslich künstlich intelligenten Problemlösungsverfahren nicht verändern oder gar aufheben, weil sie im Subjekt angelegt sind. Daraus resultiert eine Selbstverantwortung der Subjekte, die es abschliessend innerhalb einer medienpädagogisch orientierten und gleichsam orientierenden Perspektive auszuführen gilt. 


\section{Von der Problemlösung zur Urteilsfähigkeit - eine medienpädagogische Perspektive}

Erfahrungen zu machen ist das zentrale Thema des Pragmatisten Dewey und bedeutet für den Menschen, Mut aufzubringen, intelligent und kreativ zu handeln, um etwas Vertrautes in andere Formen zu bringen, neues Wissen zu generieren, Schlussfolgerungen zu ziehen und Probleme zu lösen (vgl. Dewey 1916/2011; 1916/2004). Um selbst Neues entdecken oder erfinden zu können, muss der Mensch sein Wissen und Denken einsetzen. Wissen hat im pragmatistischen Sinne einerseits die Aufgabe, zum Wirken und Handeln unter Berücksichtigung der Konsequenzen anzuleiten und ist andererseits die Bezeichnung für Ziel und Abschluss eines Urteilsfindungsprozesses. Menschliche Erkenntnis ist demnach auf die Zukunft hin orientiert, «bezieht sich auf die zukünftigen Konsequenzen des veränderten Objekts», und die Freiheit des Handelns ist durch die Kontingenz der Wirklichkeit überhaupt erst denkbar (Suhr 1994, 113). Intelligenz wird in diesem Sinne in der Handlung wirksam und mit ihr gewinnt der Mensch nicht nur die Kontrolle über eine problematische Situation, sondern sie bereichert ihn um die unmittelbare Bedeutung nachfolgender Erfahrungen (vgl. Dewey 1916/2004).

«Intelligenz ist nicht etwas, was ein für alle Mal besessen wird. Sie ist in einem fortwährenden Prozess der Bildung begriffen, und an ihr festzuhalten erfordert stete Aufmerksamkeit bei der Beobachtung der Folgen, einen aufgeschlossenen Lernwillen und Mut bei der Neuanpassung.» (Dewey 1989, 142)

Deweys Pragmatismus liefert damit eine Perspektive, die Denken als kontrollierendes «Probehandeln» und die Folge des Denkens als bessere Beherrschung einer Situation beschreibt (Suhr 1994, 49). Menschliche Intelligenz ist auf diese Weise in einen fortwährenden Lebens- und Lernprozess eingebunden, «denn schließlich wird die Anstrengung zur Kontrolle durch die Bedürfnisse, Mängel und Probleme stimuliert, die entstehen, wenn die Umwelt den Menschen bedrängt und unterdrückt» (Dewey 1916/2004, 108). Situationen repräsentieren folglich unendliche Möglichkeiten, die verborgen bleiben, wenn sie nicht durch intelligentes Handeln hervorgebracht werden. Mit anderen Worten: Im situativen Probehandeln trifft das Vollständige und Sichere stets auf das Ungewisse und Unbestimmte. Daher ist es nur konsequent zu behaupten, dass im Probehandeln das wirkmächtig wird, was sowohl der Begriff der Bildung (jede Selbstverortung in der Welt lässt auch anderes Wissen zu) als auch der Begriff des Nichtwissens (jedes Nichtwissen ebnet den Weg zu Wissen) im oben skizzierten Sinne für sich in Anspruch nehmen.

Darüber hinaus wird der Idee der Künstlichen Intelligenz mit der fortlaufenden Charakteristik des Probehandelns keine Absage erteilt. Vielmehr konzentriert sich der hier vorgebrachte Standpunkt darauf, dass der Mensch Situationen zu bewältigen hat, die zukünftig auch von Künstlicher Intelligenz mitgestaltet und verändert 
werden. Im Sinne der Freiheit seines Handelns muss er in der Lage sein, im Umgang mit intelligenten Technologien zu eigenen Entscheidungen und selbst verantwortetem, vernünftigem Handeln zu kommen (vgl. de Witt 2018). Dabei kann er durchaus den personalisierten Empfehlungen der Künstlichen Intelligenz folgen. Bei allem Komfort, die ihm die Künstliche Intelligenz bieten kann, sollte er aber im Hinblick auf seine Selbstbestimmung und -verantwortung in der Lage sein, zum einen mit den durch Technologien bedingten Kontingenzen umzugehen, zum anderen - durchaus mit ihrer Unterstützung - über Zweifel, Reflexion und Kritik neue Antworten und Problemlösungen zu finden. Kritische Urteilsfindung ist die Fähigkeit, mit Veränderungen durch Beobachten und Beurteilen von Konsequenzen auf der Grundlage von Daten, Informationen und (Nicht-) Wissen umzugehen und die konstruktive Funktion von Ideen für Entscheidungen zu nutzen. Urteilsfähigkeit zeigt sich im intelligenten bzw. verständigen Handeln, in der Formulierung von Begründungen und Argumentationslinien auf der Basis individueller Erfahrungen, die in Form von praktischen Handlungsvollzügen als experimentelle Handlungen letztlich in die Praxis umgesetzt werden. Sie ist eine der zentralen in der medienpädagogischen Praxis zu vermittelnden Bildungsaufgaben.

\section{Literatur}

Ashby, W. Ross. 1961/1981. «What is an Intelligent Machine?» In Mechanisms of Intelligence: Ross Ashby's Writings on Cybernetics, hrsg. v. Roger Conant, 295-306. Seaside, Cal: Intersystems.

Baecker, Dirk. 2002. Wozu Systeme? Berlin: Kadmos.

Baecker, Dirk. 2007. Studien zur nächsten Gesellschaft. Frankfurt a.M.: Suhrkamp.

Baecker, Dirk. 2018. 4.0 oder Die Lücke die der Rechner lässt. Berlin: Merve.

Baecker, Dirk. 2019. Intelligenz, künstlich und komplex. Berlin: Merve.

Bostrom, Nick. 2018. Die Zukunft der Menschheit. Aufsätze. Berlin: Suhrkamp.

Dewey, John. 1916/2004. «Einleitung zu den «Essays in experimenteller Logik». In Erfahrung, Erkenntnis und Wert, hrsg. und übersetzt v. Martin Suhr, 93-144. Frankfurt a.M.: Suhrkamp.

Dewey, John. 1916/2011. Demokratie und Erziehung, übers. von Jürgen Oelkers. Weinheim und Basel: Beltz.

Dewey, John. 1989. Die Erneuerung der Philosophie. Hamburg: Junius.

Görtz, Günther, und Bernhard Nebel. 2014. Künstliche Intelligenz. Frankfurt a.M.: Fischer.

Hegel, Georg Wilhelm Friedrich 1821/2015. Wissenschaft der Logik. Zweiter Band: Die subjektive Logik oder die Lehre vom Begriff. Hamburg: Felix Meiner.

Hörl, Erich. 2011. «Die technologische Bedingung. Zur Einführung». In Die technologische Bedingung. Beiträge zur Beschreibung der technischen Welt, hrsg. v. Erich Hörl, 7-53. Berlin: Suhrkamp. 
Jörissen, Benjamin, und Winfried Marotzki. 2009. Medienbildung - Eine Einführung. Bad Heilbrunn: Klinkhardt.

Koller, Hans-Christoph. 2012. Bildung anders denken - Einführung in die Theorie transformatorischer Bildungsprozesse. Stuttgart: Kohlhammer.

Krämer, Sybille. 1997. «Vom Mythos 〈Künstliche Intelligenz〉 zum Mythos «Künstliche Kommunikation > oder: Ist eine nicht-anthropomorphe Beschreibung von Internet-Interaktionen möglich?» In Mythos Internet, hrsg. v. Stefan Münker und Alexander Roesler, 83-107. Frankfurt a.M.: Suhrkamp.

Leineweber, Christian. 2020. Die Verzeitlichung der Bildung. Selbstbestimmung im technischmedialen Wandel. Bielefeld: transcript. https://doi.org/10.14361/9783839450192.

Loh, Janina. 2018. Trans- und Posthumanismus. Hamburg: Junius.

Luckin, Rosemary. 2018. Machine Learning and Human Intelligence. The future of education for the 21st century. London: UCL.

Luhmann, Niklas. 1971. «Sinn als Grundbegriff der Soziologie». In Theorie der Gesellschaft oder Sozialtechnologie - Was leistet die Systemforschung? hrsg. v. Jürgen Habermas und Niklas Luhmann, 25-100. Frankfurt a.M.: Suhrkamp.

Luhmann, Niklas. 1984. Soziale Systeme. Frankfurt a.M.: Suhrkamp.

Luhmann, Niklas. 1992. Die Wissenschaft der Gesellschaft. Frankfurt a.M.: Suhrkamp.

Luhmann, Niklas. 1995. «Die Autopoiesis des Bewußtseins». In Soziologische Aufklärung, Bd. 6 - Die Soziologie und der Mensch, hrsg. v. Niklas Luhmann 55-112. Darmstadt: Westdeutscher Verlag.

Luhmann, Niklas. 2002. Das Erziehungssystem der Gesellschaft, hrsg. v. Dieter Lenzen. Frankfurt a.M.: Suhrkamp.

Marotzki, Winfried. 1990. Entwurf einer strukturalen Bildungstheorie-Biographietheoretische Auslegung von Bildungsprozessen in hochkomplexen Gesellschaften. Weinheim: DSV.

Menke, Christoph. 2018. Autonomie und Befreiung. Studien zu Hegel. Berlin: Suhrkamp.

Mersch, Dieter. 2017. «Digital Disrupture - Digital Criticism. Für eine Kritik (algorithmischer) Vernunft». diaphanes. https://www.diaphanes.de/titel/digital-criticism-5312.

Meyer-Drawe, Käte. 2005. «Anfänge des Lernens». In Erziehung - Bildung - Negativität, hrsg. v. Dietrich Benner. Zeitschrift für Pädagogik, Beiheft 49, 24-37. Weinheim und Basel: Beltz.

Meyer-Drawe, Käte. 2007. «Kairos - Über die Kunst des rechten Augenblicks. Lutz Koch zum 65. Geburtstag». Vierteljahresschrift für wissenschaftliche Pädagogik 83(2): 241-252. https:// doi.org/10.30965/25890581-083-02-90000007.

Pongratz, Ludwig A. 1978. Zur Kritik kybernetischer Methodologie in der Pädagogik. Ein paradigmatisches Kapitel szientistischer Verkürzung pädagogisch-anthropologischer Reflexion. Frankfurt a.M.: Peter Lang.

Rohbeck, Johannes. 1993. Technologische Urteilskraft: Zu einer Ethik technischen Handelns. Frankfurt a.M.: Suhrkamp.

Rustemeyer, Dirk. 2011. Sinnformen: Konstellationen von Sinn, Subjekt, Zeit und Moral. Hamburg: Felix Meiner. 
Searle, John R. 1980. «Minds, brains and programs». Behavioral and Brain Sciences 3 (3). https://doi.org/10.1017/S0140525X00005756.

Sesink, Werner. 1990. «Künstliche Intelligenz, Systemreproduktion und Bildung». Neue Sammlung 30 (2/1990), 193-207.

Sesink, Werner. 2012. Menschliche und künstliche Intelligenz. Der kleine Unterschied. Re-Edition der Printfassung von 1993. Stuttgart: Klett-Cotta.

Simons, Herbert A. 1995. Artificial intelligence: an empirical science. Artificial Intelligence 77 (1995), 95-127.

Stehr, Nico. 1994. Arbeit, Eigentum und Wissen: Zur Theorie von Wissensgesellschaften. Frankfurt a.M.: Suhrkamp.

Steiner, George. 2006. Warum Denken traurig macht. Zehn (mögliche) Gründe. Aus dem Englischen von Nicolaus Bornhorn. Frankfurt a.M.: Suhrkamp.

Suhr, Martin. 1994. John Dewey zur Einführung. Hamburg: Junius.

Thimm, Caja. 2019. «Die Maschine - Materialität, Metapher, Mythos: Ethische Perspektiven auf das Verhältnis von Mensch und Maschine». In Die Maschine: Freund oder Feind? hrsg. v. Caja Thimm und Thomas Christian Bächle, 17-39. Wiesbaden: Springer VS. https://doi. org/10.1007/978-3-658-22954-2_2.

Wehling, Peter. 2006. Im Schatten des Wissens? Perspektiven der Soziologie des Nichtwissens. Köln: Herbert von Halem.

Wiener, Norbert. 1963. Kybernetik. Regelung und Nachrichtenübertragung im Lebewesen und in der Maschine. Düsseldorf: Econ.

Wimmer, Michael. 2014. «Antihumanismus, Transhumanismus, Posthumanismus: Bildung nach ihrem Ende». In Menschenverbesserung - Transhumanismus (Jahrbuch Pädagogik 2014), hrsg. v. Sven Kluge, Gerd Steffens und Ingrid Lohmann, 237-265. Frankfurt a.M.: Peter Lang. https://doi.org/10.3726/265764_237.

de Witt, Claudia. 2018. «Mobile Learning - Smart Learning - Next Learning. Prämissen für die Zukunft von Bildung». In Handbuch Mobile Learning, hrsg. v. Claudia de Witt und Christina Gloerfeld, 995-1014. Wiesbaden: Springer VS. https://doi.org/10.1007/978-3-658-191238_46. 